\title{
Quantification of 3D Atomic Structures and Their Dynamics by Atom-Counting from an ADF STEM Image
}

Annick De Backer ${ }^{1}$,Annelies De wael ${ }^{1}$, Ivan Lobato ${ }^{1}$, Thomas Altantzis ${ }^{1}$, Armand Béché ${ }^{1}$, Lewys Jones $^{2,3}$, Peter D Nellist ${ }^{2}$, Sara Bals ${ }^{1}$, and Sandra Van Aert ${ }^{1}$

${ }^{1 .}$ EMAT, University of Antwerp, Antwerp, Belgium

2. Department of Materials, University of Oxford, Oxford, United Kingdom

${ }^{3 .}$ CRANN \& School of Physics, Trinity College Dublin, University of Dublin, Dublin, Ireland

* Corresponding author: annick.debacker@uantwerpen.be

In order to fully exploit structure - property relations of nanomaterials, a 3D characterization at the atomic scale is often required. In recent years, the resolution of electron tomography has reached the atomic scale. However, for a successful 3D reconstruction using electron tomography, there are several limitations. Electron tomography requires multiple exposures, which is not always feasible in practice. For example, in situ experiments or the characterization of beam sensitive materials at the atomic scale are nearly impossible using electron tomography. In order to overcome these limitations, an alternative method is presented where the 3D atomic structure is reconstructed from a single projection [1-3].

The method combines atom-counting [4] in ADF STEM with prior knowledge about a material's crystal structure. The atom counts are obtained from a statistical analysis [5] of the so-called scattering crosssections, corresponding to the total intensity of electrons scattered from an atomic column. These atom counts can be used to create an initial atomic model which serves as an input for an energy minimization to obtain a relaxed 3D reconstruction of a nanostructure. This single projection reconstruction is validated against state-of-the-art compressive sensing electron tomography [3]. In Figure 1, a comparison is shown between the two atomic resolution reconstruction methods. The excellent visual match of the overall morphology of the Au nanorod demonstrates that even single image acquisitions enable us to retrieve a rather complete $3 \mathrm{D}$ picture of the underlying atomic structure.

The utility of our proposed approach is illustrated in Figure 2, where we show the quantification of the facet evolution of a Pt nanoparticle under the flow of a selected gas [6]. In a $\mathrm{H}_{2}$ environment, a more faceted surface morphology of the particles was observed with $\{100\}$ and $\{111\}$ planes being dominant. On the other hand, in a $\mathrm{O}_{2}$ environment, the percentage of $\{100\}$ and $\{111\}$ facets decreased and a significant increase of higher order facets was found, resulting in a more rounded morphology.

Such in situ experiments demonstrate that many questions in materials science require a quantification of the material's dynamics from a series of sequentially ordered images. However, the standard approach for atom-counting, where each frame is analyzed individually, does not take advantage of the time aspect available in a series of ADF STEM images. We therefore propose a new method to count the number of atoms from a time-series of ADF STEM images, using the so-called factorial hidden Markov model [7]. Scattering cross-sections resulting from a time-series of images are then considered as a dynamic network enabling us to measure changes in the 3D atomic structure in terms of transition probabilities. This new method yields far more reliable counting results than the existing atom-counting method applied to a time-series of images and is very promising for revealing atomic scale dynamics from low dose recordings [8]. 


\section{References:}

[1] S Bals et al., Nature Communications 3 (2012), p. 930.

[2] L Jones et al., Nano Letters 14 (2014), p. 6336.

[3] A De Backer et al., Nanoscale 9 (2017), p. 8791.

[4] S Van Aert et al., Physical Review B 87 (2013), p. 064107.

[5] A De Backer et al., Ultramicroscopy 134 (2013), p. 23.

[6] T Altantzis et al., Nano Letters 19 (2019), p. 477.

[7] Z Ghahramani and M I Jordan, Machine Learning 29 (1997), p. 245.

[8] This work was supported by the European Research Council (Grant 335078 COLOURATOM to SB and Grant 770887 PICOMETRICS to SVA). The authors acknowledge financial support from the Research Foundation Flanders (FWO, Belgium) through postdoctoral grants to ADB and TA, a doctoral grant to $\mathrm{ADw}$, and project fundings.

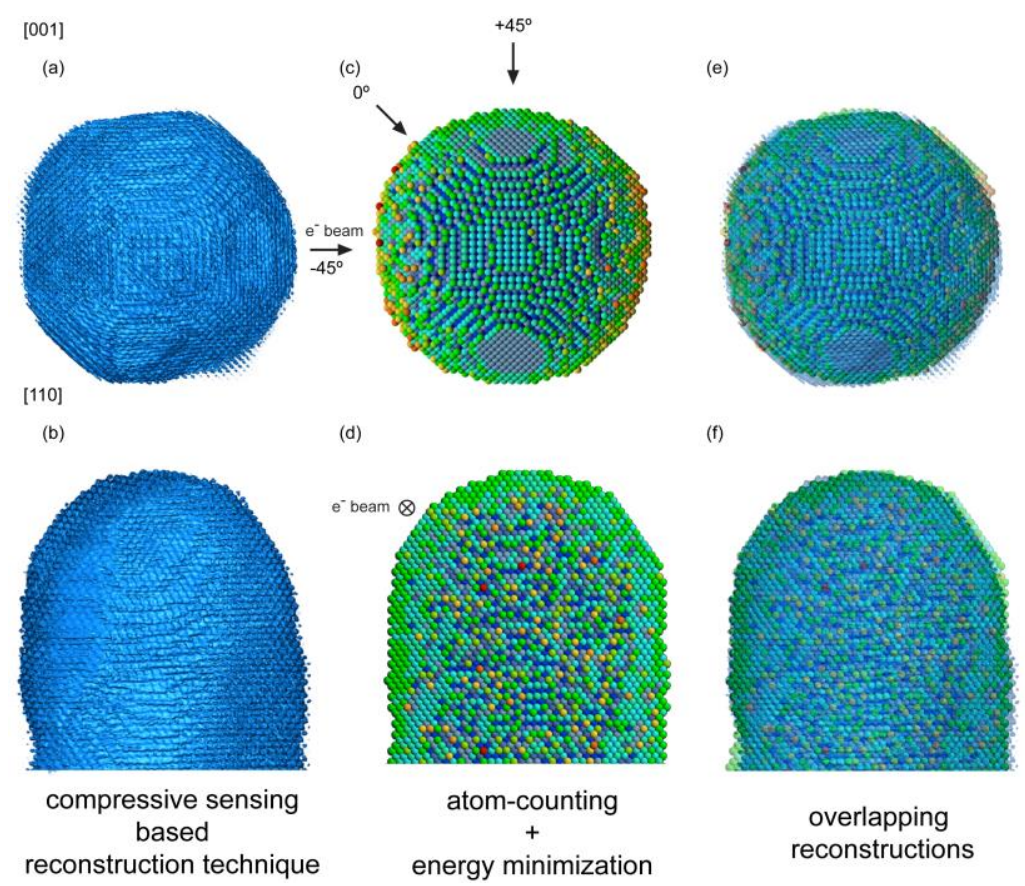

Figure 1. Comparison of the atom-counting/energy minimization and atomic resolution tomography reconstruction. (a,b) Compressive sensing based reconstruction of a Au nanorod viewed along [001] and [110] direction. (c,d) Reconstruction based on atom-counting and energy minimization using a single projection image. (e,f) Overlap of the reconstructions shown in $(\mathrm{a}, \mathrm{c})$ and $(\mathrm{b}, \mathrm{d})[3]$.

(a)

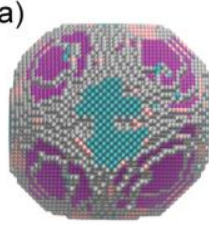

$5 \% \mathrm{H}_{2}$ in $\mathrm{Ar}$

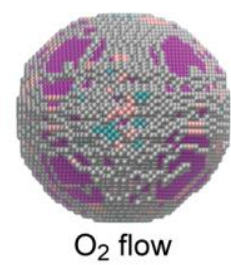

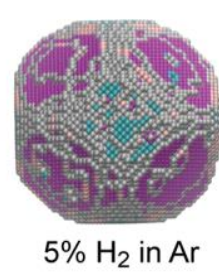
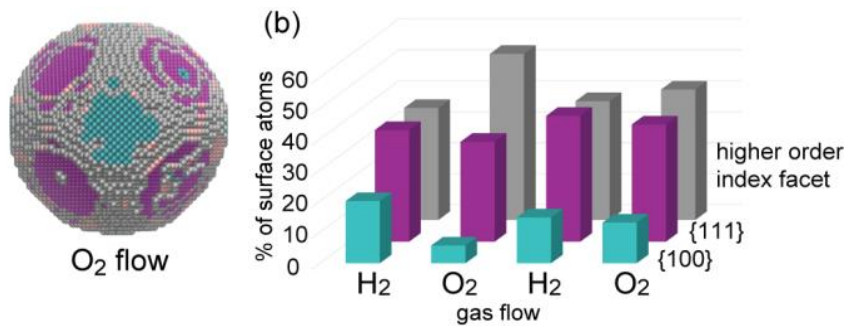

Figure 2. (a) Structural evolution of a Pt nanoparticle under different environmental conditions. (b) Occurrence of different surface facets as a function of the gas flow in time [6]. 